UDC 669.295:621.785

DOI: 10.15587/2706-5448.2021.237250

Article type «Reports on Research Projects»

\section{Vasyl Trush, Alexander Luk'yanenko, Viktor Fedirko}

\title{
STUDY OF TITANIUM OXIDATION KINETICS AT TEMPERATURE ABOVE POLYMORPHIC TRANSFORMATION
}

The object of research is the analytical description of the phenomena in the near-surface layer, which are caused by the interaction of titanium with oxygen at high temperatures. These are temperatures that exceed the polymorphic transformation of the metal. High-temperature oxidation gives titanium products unique performance properties. Of course, such characteristics are determined, first of all, by the state of the near-surface layer. Therefore, an understanding of oxidation processes will make it possible to predict the state of the near-surface layer after heat treatment. However, to date, no unified approach has been created to describe the mechanism and kinetics of high-temperature oxidation of titanium in the near-surface layer. Indeed, most of the existing approaches make it possible to predict the nature of oxidation in the bulk of the metal. Some scientific papers describe the kinetics of oxidation, taking into account only the formation and growth of oxide layers. However, simultaneously with oxide formation, a diffusion zone is formed, which significantly affects the kinetics. Therefore, today one of the most problematic areas of high-temperature titanium oxidation is the description of the processes that take place in the near-surface layer.

In this work, to describe the kinetics of high-temperature oxidation of titanium, in addition to the formation and growth of the oxide layer, the formation and growth of the diffusion zone is taken into account. In the diffusion zone, under the influence of structural phase transformations, solid solutions of oxygen are formed in the alpha and beta phases. This approach made it possible to take into account additional factors and thereby more accurately describe the processes of high-temperature oxidation of titanium. As a result of the calculations, the thickness of the oxide layer of the diffusion zone is given depending on the oxygen concentration and the duration of treatment. And also the dependences of the kinetics of displacement of the boundary of the oxide-diffusion layer are given and a system of equations for calculating the ratio of the formed phase components is developed. Thanks to the proposed analytical approach, it will be possible to calculate the sizes of interphase boundaries on the basis of temperature-time parameters and oxygen concentration and thereby form a hardened near-surface layer with certain functional properties.

Keywords: oxygen diffusion in titanium, high-temperature oxidation of titanium, kinetics of displacement of interphase boundaries.

Trush, V., Luk'yanenko, A., Fedirko, V. (2021). Study of titanium oxidation kinetics at temperature above polymorphic transformation. Technology Audit and Production Reserves, 4 (1 (60)), 37-41. doi: http://doi.org/10.15587/2706-5448.2021.237250

\section{Introduction}

Titanium and titanium alloys are promising structural materials for products of modern aviation and space technology, as well as for medical purposes [1-3]. The complex of characteristics of products made of these materials essentially depends on the properties of their surface layer [4-6]. An effective method of strengthening them is thermal diffusion saturation with elements of embodiment, in particular, nitrogen, oxygen or carbon [7-9]. The interaction of titanium with these elements at high temperatures is accompanied not only by the formation and growth of a nitride, oxide or carbide film, but also by a significant dissolution of nitrogen, oxygen and carbon in the metal. The competition of these processes significantly complicates the study of the kinetics and mechanism of such interaction. Useful information on this issue can be provided by the results of studies of the high-temperature interaction of titanium with nitrogen, oxygen, and carbon. At the same time, such treatments are of practical interest, since they allow the formation of deep diffuse layers without a continuous phase film on the titanium surface [10].

In the change in the properties of the near-surface layers of structural materials in many cases, then during their chemical-thermal treatment or under operating conditions at high temperatures, diffuse processes are decisive. Interesting for such studies, both theoretically and experimentally, is, in particular, the study of the high-temperature interaction of titanium with oxygen. Indeed, titanium at a temperature of $T_{\alpha \Leftrightarrow \beta}=882{ }^{\circ} \mathrm{C}$ undergoes a polymorphic 
transformation, as a result of which there is a change in the crystal lattice from a hexagonal close-packed (HCP) to a body-centered cubic (BCC).

Oxygen in relation to titanium of new alloys has a high chemical affinity, as a result of which an oxide layer forms and grows on the surface. At the same time, oxygen is $\alpha$-stabilizer that can stimulate structural transformations in titanium [9]. However, the work did not take into account the role of the embodiment element itself in structural transformations as $\alpha$-stabilizer. Therefore, it is urgent to develop analytical calculations to determine the thickness of the oxide and diffusion layers. Thus, the object of research is the analytical description of the phenomena in the near-surface layer caused by the interaction of titanium with oxygen at high temperatures. The aim of this research is to analytically reveal the kinetic features of the formation of the oxide and diffusion layers of titanium at temperatures above the polymorphic transformation.

\section{Methods of research}

In studying the assessment of the influence of the temperature-time parameters of the diffusion saturation of titanium with oxygen on the formation of the diffusion zone, the solutions of the Fick equation were used. The calculation of the oxygen diffusion coefficients for temperatures was made according to the Arrhenius dependences. For an analytical description of oxidation, some model assumptions have been made. In particular, the fact that the oxide film forms instantly on the titanium surface at zero time, and the oxygen concentration on the surface does not change with time and corresponds to the stoichiometric titanium oxide $\left(\mathrm{TiO}_{2}\right)$. And also the fact that constant oxygen concentrations are maintained at the interphase boundaries, which correspond to equilibrium concentrations.

\section{Research results and discussion}

According to the phase diagram (Fig. 1), titanium undergoes a polymorphic transformation (a change in the crystal lattice from HCP to BCC) at $T_{\alpha \Leftrightarrow \beta}=882{ }^{\circ} \mathrm{C}$ [11]. The work focuses on the high-temperature $\left(T>T_{\alpha \Leftrightarrow \beta}\right)$ interaction of titanium with oxygen. At the initial stage of the titanium oxidation process, oxygen atoms penetrate into the crystal lattice of the metal, which results in the formation of a solid solution embodiment based on oxygen $\operatorname{Ti}(\mathrm{O})$. With further saturation of titanium with oxygen in an amount exceeding the maximum solubility, a new phase appears in the system based on a stable $\mathrm{TiO}$ compound.

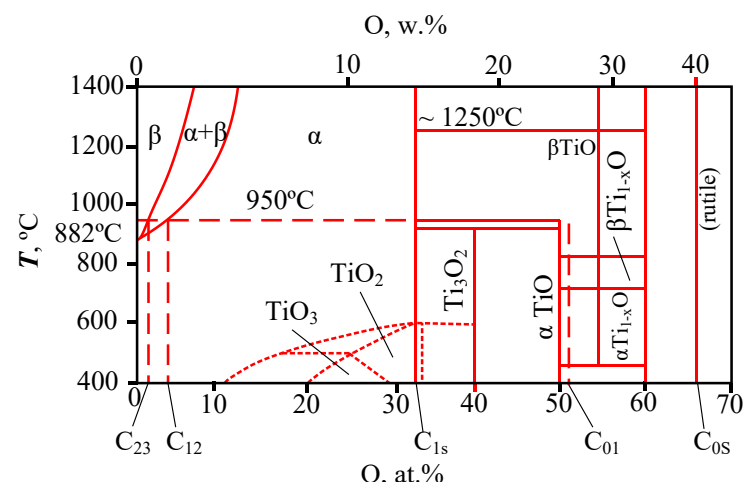

Fig. 1. Diagram of the state of the system «Ti-0» [1]
Let's note that the solubility of oxygen in the $\alpha$-phase is rather high compared to the $\beta$-phase. At the same time, their diffusion coefficients in the $\alpha$-phase are two orders of magnitude lower than in the $\beta$-phase $[1,2]$.

Phenomenological model. First of all, let's consider the process of isothermal thermal diffusion saturation of titanium with oxygen at a temperature exceeding the temperature of the polymorphic transformation $\left(T>T_{\alpha \Leftrightarrow \beta}\right)$. So, then lets get that the initial microstructure of the above metal has a $\beta$-phase. For thermodynamic analysis, a diagram of a gas-saturated titanium layer is proposed (Fig. 2).

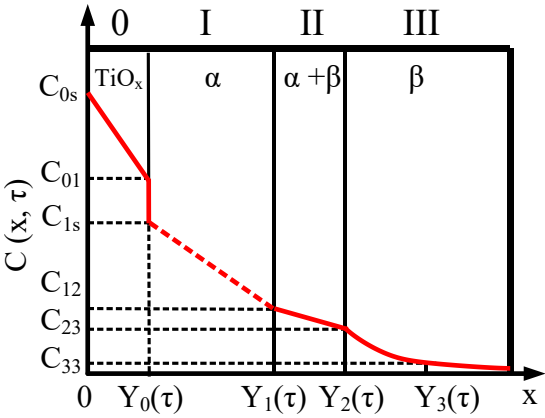

Fig. 2. Scheme of oxygen concentration distribution during titanium saturation at $T>T_{\alpha \Leftrightarrow \beta}$

As a result of the interaction of titanium with an oxygen-containing medium, not only the formation of an oxide layer $\left(0,0<x<Y_{0}(\tau)\right.$ zone $)$ takes place, but also a diffusion zone. The diffuse zone is three-layer. The first layer $\left(I, Y_{0}(\tau)<x<Y_{1}(\tau)\right)$ zone), which is in contact with the oxide layer, is an $\alpha$-solid solution significantly enriched in oxygen due to its high solubility in the $\alpha$-phase of titanium. Such a layer forms and grows upon saturation due to the dissolution of oxygen and structural transformations in titanium. The layer (zone III, $Y_{2}(\tau)<x<\infty$ ), which is adjacent to the base at the saturation temperature and represents the $\beta$-phase of titanium enriched with oxygen. Between the first and third layers, a transition zone of ionizing radiation is formed and grows $\left(Y_{1}(\tau)<x<Y_{2}(\tau)\right)$, which is a dispersed mixture of solid solutions of oxygen in the $\alpha$ - and $\beta$-phases of titanium.

Before an analytical description of titanium oxidation, it is necessary to make some model assumptions. Since the purpose of the chemical-thermal treatment of titanium samples is to strengthen their surface layers first, the object of analytical study of the titanium saturation kinetics is the half-space $(0 \leq x<\infty)$. An oxide film is formed instantly on the titanium surface at zero time, the surface oxygen concentration does not change with time and corresponds to the stoichiometric titanium oxide $\left(\mathrm{TiO}_{2}\right)$. At the interphase boundaries, constant oxygen concentrations are maintained, which correspond to equilibrium concentrations (Fig. 1). The thermal diffusion process in the aforementioned inhomogeneous system will be described using the Fick system of equations:

$$
D_{i} \partial^{2} C_{i}(x, \tau) / \partial x^{2}=\partial C_{i}(x, \tau) / \partial \tau, i=0,1,2,3,
$$

where $C_{i}(0, \tau)$ and $D_{i}$ - concentration and diffusion coefficients of oxygen in the corresponding layers; the index $i=0$ corresponds to the oxide layer $\mathrm{TiO}_{2-x}\left(0<x<Y_{0}(\tau)\right)$, $i=1$ - to the zone $(\alpha+\beta)$-Ti $\left(Y_{1}(\tau)<x<Y_{2}(\tau)\right), i=2-\beta$-Ti zone $\left(Y_{2}(\tau)<x<Y_{3}(\mu)\right) ; x$ - spatial coordinate, $\tau$ - time. 
Initial conditions $(\tau=0)$ :

$$
C_{i}(x, \tau)=0, Y_{i}(0)=0 \text {, for } x>0 .
$$

Limit conditions $(\tau>0)$ :

$$
\begin{aligned}
& C_{0}(0, \tau)=C_{0 s}, C_{3}(\infty, \tau)=0, C_{0}\left(Y_{0}(\tau), \tau\right)=C_{01}, \\
& C_{01}\left(Y_{0}(\tau), \tau\right)=C_{1 s}, C_{1}\left(Y_{1}(\tau), \tau\right)=C_{2}\left(Y_{1}(\tau), \tau\right)=C_{12}, \\
& C_{1}\left(Y_{2}(\tau), \tau\right)=C_{3}\left(Y_{12}(\tau), \tau\right)=C_{23}
\end{aligned}
$$

The motion of the interfacial boundaries is given by parabolic dependences:

$$
\begin{aligned}
& Y_{0}(\tau)=2 \beta_{0} \sqrt{D_{0} \tau}, Y_{1}(\tau)=2 \beta_{01} \sqrt{D_{1} \tau}, \\
& Y_{2}(\tau)=2 \beta_{021} \sqrt{D_{12} \tau} .
\end{aligned}
$$

It should be noted that here $\beta_{j}(j=0,1,2)$ are dimensionless constants (for a specific saturation temperature), which let's determine from the law of conservation of mass at the interfacial boundaries. Therefore, for constant flows at the interfacial boundaries $Y_{j}(\tau)$ :

$$
\begin{aligned}
& -\left.D_{0} \frac{C_{0}}{x}\right|_{x=Y_{0(\tau)-0}}+\left.D_{0} \frac{C_{1}}{x}\right|_{x=Y_{0(\tau)+0}}=\left(C_{01}-C_{0 s}\right) \frac{d Y_{1}(\tau)}{d \tau},
\end{aligned}
$$

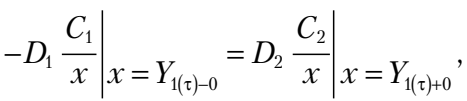

$$
\begin{aligned}
& \left.D_{2} \frac{C_{2}}{x}\right|_{x=Y_{2(\tau)-0}}=\left.D_{3} \frac{C_{3}}{x}\right|_{x=Y_{2(\tau)+0}} .
\end{aligned}
$$

It is rather difficult to obtain a solution to the diffusion problem (1)-(5) in an analytical form. Therefore, let's use the method of approximate solution of this problem. Let's accept linear laws of the distribution of oxygen concentrations in the oxide layer and in the first two layers of the diffusion zone, corresponding to a quasi-stationary state, and the Gaussian law for the distribution of oxygen in the third layer:

$$
\begin{aligned}
& C_{0}(0, \tau)=C_{0 s}-\left(C_{0 s}-C_{01}\right) \frac{x}{Y(\tau)}, \\
& C_{10}(0, \tau)=C_{1 s}-\left(C_{1 s}-C_{12}\right) \frac{x-Y_{0}(\tau)}{Y_{1}(\tau)-Y_{0}(\tau)}, \\
& C_{2}(0, \tau)=C_{12}-\left(C_{12}-C_{23}\right) \frac{x-Y_{1}(\tau)}{Y_{2}(\tau)-Y_{1}(\tau)}, \\
& C_{3}(x, \tau)=C_{23} \operatorname{erfc} \frac{x-Y_{2}(\tau)}{2} \sqrt{D_{3}} \frac{x-Y_{0}(\tau)}{Y_{1}(\tau)-Y_{0}(\tau)} .
\end{aligned}
$$

The selected functions $C_{j}(x, \tau)$ satisfy the initial and limiting (2) and limiting (1) conditions, as well as the differential equation (1). The use of the conjugation condition at the interphase boundaries (5), as well as relation (4), makes it possible to obtain such a system of transcendental equations for calculating the parameters $\beta_{j}(i=1,2,3)$ :

$$
\begin{aligned}
& \frac{A_{0}}{2 \beta_{0}}\left[\frac{1}{\beta_{0}}-\frac{1}{A_{1} y_{0}\left(\beta_{1}-\beta_{0} y_{0}\right)}\right]=1, \\
& \frac{\beta_{1}-\beta_{0} y_{0}}{A_{2} y_{0}\left(\beta_{2}-\beta_{1} y_{1}\right)}=1, \frac{2\left(\beta_{2}-\beta_{1} y_{01}\right)}{A_{3} y_{2} \pi}=1 .
\end{aligned}
$$

Having solved the system of equation:

$$
\begin{aligned}
& \beta_{0}=A_{0}\left[\sqrt{B^{2}+2 / A_{0}}-B\right] / 2, \\
& \beta_{1}=\beta_{0} y_{0}+A_{2} A_{3} y_{1} y_{2} \sqrt{\pi / 2}, \\
& \beta_{2}=\beta_{1} y_{1}+A_{3} y_{2} \sqrt{\pi / 2}, \\
& B=1 /\left[A_{1} A_{2} A_{1} y_{0} y_{1} y_{1} \sqrt{\pi}\right], \\
& y_{0}=\sqrt{D_{0} / D_{1}}, y_{1}=\sqrt{D_{1} / D_{2}}, y_{2}=\sqrt{D_{2} / D_{3}},
\end{aligned}
$$

where

$$
\begin{aligned}
& A_{0}=\frac{C_{0 s}-C_{01}}{C_{01}-C_{0 s}}, A_{1}=\frac{C_{0 s}-C_{01}}{C_{1 s}-C_{12}}, \\
& A_{2}=\frac{C_{1 s}-C_{12}}{C_{12}-C_{23}}, A_{0}=\frac{C_{12}-C_{23}}{C_{23}} .
\end{aligned}
$$

It should be noted that the parameter $\beta_{j}$ depends on the values of the oxygen concentrations at the interphase boundaries and the diffusion coefficients, which, in turn, depend on the saturation temperature. Taking the values of these parameters, in particular, corresponding to the temperature $T=950{ }^{\circ} \mathrm{C}$, in accordance with relations (8), constants $\beta_{j}(j=0,1,2)$ were calculated: $\beta_{0}=0.082 ; \beta_{1}=1.481$; $\beta_{1}=0.789$. For the calculations, the following values of the diffusion coefficients were used: $D_{0}=D_{\mathrm{TiO}_{2}}=2.5 \cdot 10^{-11} \mathrm{~cm}^{2} / \mathrm{s}$, $D_{1}=D_{\alpha}=2.1 \cdot 10^{-9} \mathrm{~cm}^{2} / \mathrm{s}, D_{2}=10 \cdot D_{1} \mathrm{~cm}^{2} / \mathrm{s}$ (assuming that $D_{1}<D_{2}<D_{3}, D_{3}=1.6 \cdot 10^{-7} \mathrm{~cm}^{2} / \mathrm{s}$, and such values of oxygen concentrations at the interphase boundaries: $C_{0 s}=66 \% \mathrm{~atm}$, $C_{1 s}=33 \%$ atm, $C_{12}=2 \%$ atm., Fig. 3 ).

Further, taking into account relation (4), let's represent the motion of the interphase boundaries as follows:

$$
Y_{0}(\tau)=K_{0} \sqrt{\tau}, Y_{1}(\tau)=K_{1} \sqrt{\tau}, Y_{2}(\tau)=K_{2} \sqrt{\tau},
$$

where $K_{0}=2 \beta_{0} \sqrt{D_{0}}, K_{1}=2 \beta_{1} \sqrt{D_{1}}, K_{2}=2 \beta_{2} \sqrt{D_{2}}$ - the constants of parabolic growth of the corresponding oxide layer and oxygen-stabilized $\alpha-,(\alpha+\beta)$-sections of the diffusion zone. In particular, for the saturation temperature $T=950^{\circ} \mathrm{C}$, these constants have the following meanings:

$$
\begin{aligned}
& K_{0}=8.2 \cdot 10^{-7} \mathrm{~cm} / \mathrm{s}^{-2}, K_{1}=1.35 \cdot 10^{-4} \mathrm{~cm} / \mathrm{s}^{-2}, \\
& K_{2}=2.3 \cdot 10^{-4} \mathrm{~cm} / \mathrm{s}^{-2} .
\end{aligned}
$$

Based on the constants of the parabolic growth of layers (10) and the dependence of the motion of the interphase boundaries (9), the kinetics of the displacement of the interphase boundaries was calculated (Fig. 3, $a, b$ ). Also, such information makes it possible to establish changes in the thickness of the oxide layer (Fig. 3, a) and $\alpha,(\alpha+\beta)$ and $\beta$-regions of the diffusion zone (Fig. 3,c). The sizes of the oxide layer and different areas of the diffusion zone can be represented by the corresponding ratios:

$$
\begin{aligned}
& \Delta Y_{0}(\tau)=Y_{0}(\tau), \Delta Y_{1}(\tau)=Y_{1}(\tau)-Y_{0}(\tau)=\widetilde{K_{1}} \sqrt{\tau}, \\
& \Delta Y_{2}(\tau)=Y_{2}(\tau)-Y_{1}(\tau)=\widetilde{K_{2}} \sqrt{\tau}
\end{aligned}
$$

where $\widetilde{K_{1}}=K_{1}-K_{0}, \widetilde{K_{2}}=K_{2}-K_{1}$.

It should be noted that the $\beta$-region of the diffusion zone is recognized by moving the conventional boundary with a specific oxygen concentration, for example, $C_{33}=0.25$ at. $\%$. 

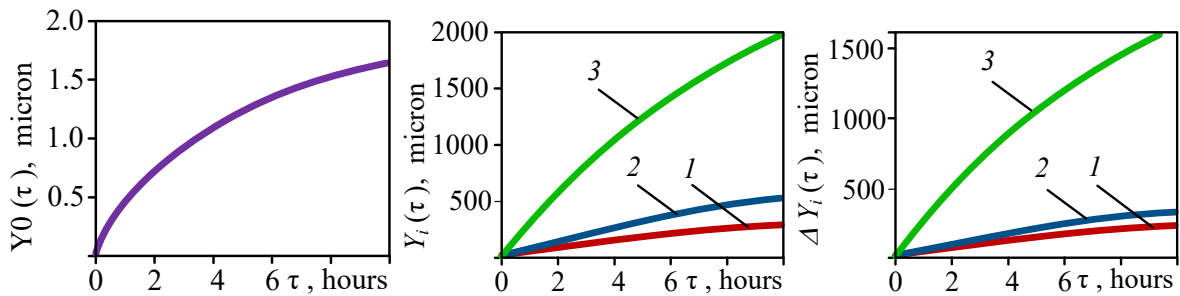

Fig. 3. Kinetics of displacement of interphase boundaries:

$a$ - limit of the oxide layer $Y_{0}(\tau) ; b-Y_{1}(\tau) ; 2-Y_{2}(\tau) ; 3-Y_{3}(\tau)$ and changes in the thickness of the layers;

$c-1$ - thickness of the $\alpha$-zone, 2 - thickness of the $(\alpha+\beta)$-zone, 3 - thickness of the $\beta$-zone during titanium oxidation at $T=950{ }^{\circ}[$

Based on relations (6), the concentration profiles of oxygen in the gas-saturated zone of titanium after oxidation for 5 and 10 hours were calculated (Fig. 4). Of course, the displacements of the interphase boundaries (Fig. 3, $a, b$ ) and the increase in the thickness of various sections of the diffusion zone (Fig. 3,c) with an increase in the holding time occur according to parabolic dependences. However, it should be taken into account that the change in parabolic dependences occurs in proportion to the corresponding constants of parabolic growth $K_{j}$.

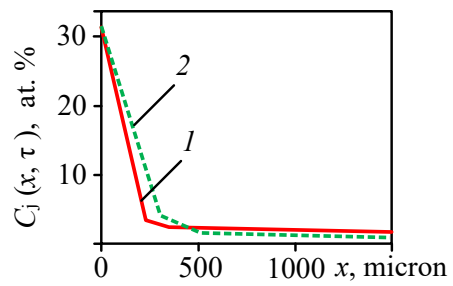

Fig. 4. Distribution of axygen in the diffusion zone of titanium after oxidation at $T=950{ }^{\circ} \mathrm{C}: 1-5 \mathrm{~h} ; 2-10 \mathrm{~h}$

The diffusion coefficient and the amount of dissolved oxygen affect the thickness of the layers of the $\alpha$-phase, oxide layer, $\beta$-phase. The diffusion coefficients of oxygen in the $\beta$-phase are $2-4$ orders of magnitude higher than the diffusion coefficients in the $\alpha$-phase and the oxide layer. Therefore, the thickness of the $\beta$ region is much greater than the thickness of other sections of the modified gassaturated zone (Fig. 3)

The distribution of oxygen in the diffusion zone is affected by its different solubility in the $\alpha$ - and $\beta$-phases. If phase-structural transformations did not occur in the diffusion zone, then the oxygen profile in this zone would have a low gradient due to insignificant solubility in the $\beta$-phase. According to the literature and scientific data, oxygen, being an $\alpha$-stabilizer, significantly stimulates the phase-structural $\beta \leftrightarrow \alpha$ transformation of titanium in the region of the diffusion zone adjacent to the oxide layer. And since the solubility of oxygen in the $\alpha$-phase is much higher than in the $\beta$-phase, it is possible to confidently say that in the I zone the concentration distribution of the oxygen component in the near-surface titanium layer will have a significant gradient (Fig. 4).

\section{Conclusions}

In the course of an analytical study, based on taking into account the structural phase transitions in the nearsurface layer, regularities were obtained that determine the size of the oxide and diffusion layers on titanium after high-temperature oxidation. A system of equations has been developed that takes into account various laws of the distribution of oxygen concentration in the oxide layer and diffuse zones and determines their ratio. The dependence of the motion of the interphase boundaries between the oxide layer and the diffuse layer is calculated analytically, which takes into account not only the diffusion coefficients, but also the solubility of oxygen.

The constants of the parabolic growth of the layers were calculated, which made it possible to calculate the oxygen distribution in the near-surface layer at $T=950{ }^{\circ} \mathrm{C}$ for time holdings of 5 hours and 10 hours. It is shown that the calculation of the oxygen concentration profiles in the diffusion zone of titanium at $T=950{ }^{\circ} \mathrm{C}$ for different time periods must necessarily include both the constants of the parabolic growth of the layers and the oxygen solubility in the $\alpha$-phase.

The results of the research will be useful for thermists when choosing the temperature-time parameters of the high-temperature treatment of titanium.

\section{References}

1. Lutjering, G., Williams, J. C. (2007). Titanium. Springer-Verlag Berlin Heidelberg, 442. doi: http://doi.org/10.1007/978-3-54073036-1

2. Moiseyev, V. N. (2006). Titanium Alloys. Russian aircraft and aerospace applications. London-New York-Singapore: Taylor \& Francis Group, 207.

3. Hideki, F., Kazuhiro, T., Yoshito, Y. (2003). Application of titanium and its alloys for automobile parts. Nippon Steel Technical Report, 88, 70-75.

4. Dong, H., Li, X. Y. (2000). Oxygen boost diffusion for the deep-case hardening of titanium alloys. Materials Science and Engineering: A, 280 (2), 303-310. doi: http://doi.org/10.1016/ s0921-5093(99)00697-8

5. Guleryuz, H., Cimenoglu, H. (2005). Surface modification of a Ti-6Al-4V alloy by thermal oxidation. Surface and Coatings Technology, 192 (2-3), 164-170. doi: http://doi.org/10.1016/ j.surfcoat.2004.05.018

6. Ebrahimi, A. R., Zarei, F., Khosroshahi, R. A. (2008). Effect of thermal oxidation process on fatigue behavior of Ti-4Al-2V alloy. Surface and Coatings Technology, 203 (3-4), 199-203. doi: http://doi.org/10.1016/j.surfcoat.2008.08.038

7. Pohrelyuk, I. M., Sheykin, S. E., Dub, S. M., Mamalis, A. G., Rostotskii, I. Y., Tkachuk, O. V., Lavrys, S. M. (2016). Increasing of functionality of c.p. titanium/UHMWPE tribo-pairs by thermodiffusion nitriding of titanium component. Biotribology, 7, 38-45. doi: http://doi.org/10.1016/j.biotri.2016.08.002

8. Yaskiv, O. I. (2008). Surface hardening of titanium by noncontact thermodiffusion carbonitriding. Materials Science, 44 (5), 659-664. doi: http://doi.org/10.1007/s11003-009-9135-1

9. Malinov, S., Zhecheva, A., Sha, W. (2003). Modelling the nitriding in titanium alloys. ASM International: Materials Park, 344-352.

10. Fedirko, V. M., Luk'yanenko, O. H., Trush, V. S. (2014). Influence of the Diffusion Saturation with Oxygen on the Durability and Long-Term Static Strength of Titanium Alloys. Materials Science, 50 (3), 415-420. doi: http://doi.org/10.1007/ s11003-014-9735-2 
11. Oshida, Y. (2007). Bioscience and bioengineering of titanium materials. Elsevier, 430. doi: http://doi.org/10.1016/b978-008-045142-8.x5000-2

$\triangle$ Vasyl Trush, PhD, Department of Materials Science of Surface Engineering, Karpenko Physico-Mechanical Institute of the National Academy of Sciences of Ukraine, Lviv, Ukraine, ORCID: https:// orcid.org/0000-0002-2264-3918, e-mail: trushvasyl@gmail.com

Alexander Luk'yanenko, PhD, Department of Materials Science of Surface Engineering, Karpenko Physico-Mechanical In- stitute of the National Academy of Sciences of Ukraine, Lviv, Ukraine, ORCID: https://orcid.org/0000-0001-6642-2300, e-mail: AGNSLukyanenko@I.UA

Viktor Fedirko, Doctor of Technical Sciences, Professor, Department of Materials Science of Surface Engineering, Karpenko PhysicoMechanical Institute of the National Academy of Sciences of Ukraine, Lviv, Ukraine, ORCID: https://orcid.org/0000-0002-4337-1691, e-mail: fedirko@ipm.lviv.ua

$\triangle$ Corresponding author 